\title{
The Quadratic-Form Representation of the Pre-Averaging Estimator
}

\begin{abstract}
Selma Chaker
Bank of Canada

Volatility forecasts are central to many financial issues, including empirical asset pricing finance and risk management. In this paper, I derive a new quadratic-form representation of the pre-averaging volatility estimator of Jacod et al. (2009), which allows for the theoretical analysis of its forecasting performance.

Keywords: Realized volatility, market microstructure noise, eigenfunction stochastic volatility models, Mincer-Zarnowitz regression.
\end{abstract}

\section{Introduction}

Measuring volatility using high-frequency data has attracted growing interest since the late 1990s, for many reasons. First, thanks to the increased availability of large data samples, we can observe almost continuous data processes, which in turn justify the use of the continuous time framework. The Trades and Quotes (TAQ) database ${ }^{1}$ usually releases one-second frequency prices and quotes, but recently it has been releasing one-millisecond frequency data. Such an ultra-high-frequency data set opens up research opportunities to explore intraday volatility features and spot volatility estimation. The second major reason for the growing interest is that the model-free approach of the theory of quadratic variation is not vulnerable to model misspecification, as is the case with other approaches from the parametric literature.

The realized variance, which is the sum of the squared intraday returns, would be a consistent estimator of the integrated variance if there were no frictions in the market (see Jacod (1994), and Barndorff-Nielsen and Shephard (2002) for a proof of the consistency and the limit theory of the realized volatility). However, observed high frequency prices are contaminated with frictions or market microstructure noise such as bid-ask bounce and transaction costs. Consequently, the realized variance is not anymore a consistent estimator of the integrated variance.

Many robust-to-noise volatility estimators have been derived; see, for example, Zhang et al. (2005), Zhang (2006), Barndorff-Nielsen et al. (2008), and Jacod et al. (2009). Early assumptions hypothesized an exogenous independent and identically distributed (i.i.d.) dynamic for the noise. Later on, this assumption was relaxed to allow for some forms of endogeneity with the frictionless price and an autocorrelated noise. More specifically, the pre-averaging estimator of Jacod et al. (2009) is robust to heteroscedastic market microstructure noise and achieves the optimal rate of convergence of $N^{1 / 4}$, where $N$ is the size of the high frequency data sample.

\footnotetext{
1 The TAQ database is a collection of intraday trades and quotes for all securities listed on the New York Stock Exchange, American Stock Exchange, Nasdaq National Market System and SmallCap issues. TAQ provides historical tick-by-tick data of all stocks listed on NYSE back to 1993.
} 
In this paper, I provide a mathematically tractable representation of the pre-averaging estimator of the integrated volatility. This quadratic-form representation is particularly useful for deriving closed-form expressions to measure the volatility forecasting performance under an eigenfunction stochastic volatility (ESV) framework. The models of the ESV class were developed by Meddahi (2001) for standard i.i.d. market microstructure noise. To theoretically examine the performance of volatility estimators in terms of forecasting, Andersen et al. (2011) use the ESV framework. Andersen et al. (2004) and Sizova (2011) also rely on the ESV representation.

The rest of the paper is structured as follows. In the next section, I provide the notation and describe the setting of volatility estimators. Section 3 states the quadratic-form representation for the pre-averaging estimator of integrated variance. In Section 4, I apply this new representation to the measurement of a volatility forecasting problem in an ESV framework. The last section concludes.

\section{Notation and Setting}

This section describes the theoretical framework of the volatility estimators.

The logarithm of the frictionless price is denoted by $p_{s}^{*}$ and evolves as a continuous semimartingale given by

$$
d p_{s}^{*}=\sigma_{s} d W_{s}, s \in[0, T],
$$

where $W_{s}$ is a Wiener process and $\sigma_{s}$ is a càdlàg volatility function. By assumption, the drift term is zero and $W_{s}$ and $\sigma_{s}$ are independent to exclude leverage and drift effects. These simplifying assumptions could be relaxed using the ESV framework. Andersen et al. (2006) provide a starting point for a direct analytical exploration and quantification of such effects in the case of white noise.

The object of interest is the integrated variance of the latent price $p_{s}^{*}$ given by

$$
I V_{t+1}=\int_{t}^{t+1} \sigma_{s}^{2} d s
$$

I assume the usual additive-form contamination for the observed log price $p_{s}$,

$$
p_{s}=p_{s}^{*}+u_{s}
$$

where $u_{s}$ is the market microstructure noise which is also latent. The standard assumption on the noise is that $u_{s}$ is i.i.d. and independent from the frictionless price $p_{s}^{*}$. Heteroscedasticity in the noise is accounted for in, among other, Kalnina and Linton (2008), Barndorff-Nielsen et al. (2011), and Jacod et al. (2009). The two time scales estimator of Zhang et al. (2005), derived under the standard assumption for the noise, is extended to the multi time scales estimator of Ait-Sahalia et al. (2011) to allow for serial correlation in the noise.

\section{The Realized Volatility}

A consistent estimator for the integrated variance when there is no market microstructure noise is the traditional realized volatility, given by

$$
R V_{t}^{*}(h)=\sum_{i=1}^{1 / h} r_{t-1+i h}^{* 2}
$$

where $h=1 / N$ and $r_{s}^{*}=p_{s}^{*}-p_{s-h}^{*}$. In practice, we do observe the intraday returns $r_{s}=p_{s}-p_{s-h}$. The contaminated and frictionless returns are linked as $r_{s}=r_{s}^{*}+e_{s}$, where $e_{s}=u_{s}-u_{s-h}$. The feasible realized variance based on high-frequency returns, thus, is 


$$
R V_{t}(h)=\sum_{i=1}^{1 / h} r_{t-1+i h}^{2}
$$

The realized volatility is inconsistent for integrated volatility because of the noise. In the next subsection, however, the expression of the pre-averaging estimator is consistent for the integrated variance in the presence of market microstructure noise.

\section{The Pre-averaging Estimator}

To mitigate the effect of the noise, the pre-averaging estimator averages intraday returns. Formally, this robust-to-noise volatility estimator is given by

$$
R M_{t}^{\text {pre }}=\frac{12}{\theta \sqrt{N}} \sum_{i=0}^{N-k}\left\{\sum_{j=1}^{k} \Phi\left(\frac{j}{k}\right) r_{t-1+(i+j) h}\right\}^{2}-\frac{6}{\theta^{2} N} \sum_{i=1}^{N} r_{t-1+i h}^{2}
$$

where $\frac{k}{\sqrt{N}}=\theta+\mathcal{O}\left(N^{-1 / 4}\right)$ for some $\theta>0$ and $\Phi(x)=\min (x, 1-x)$. The tuning parameters of the pre-averaging estimator are $\theta$, the function $\Phi($.$) and the integer k.$

\section{The Quadratic-form Representation}

To facilitate the analysis of the realized measures $R M_{t}$ of volatility, whether traditional or robust-to-noise, I use the quadratic-form representation. For a sampling frequency $h$ of intraday returns, this representation is given by

$$
R M_{t}(h)=\sum_{1 \leq i, j \leq 1 / h} q_{i j} r_{t-1+i h} r_{t-1+j h}
$$

For example, $R V_{t}(h)$ could be written as

$$
R V_{t}(h)=R V_{t}^{a l l}(h)=\sum_{1 \leq i, j \leq 1 / h} q_{i j}^{a l l} r_{t-1+i h} r_{t-1+j h}
$$

where $q_{i j}^{a l l}=1$ if $i=j$ and $q_{i j}^{a l l}=0$ otherwise.

I show that the pre-averaging estimator could also be represented in a quadratic form:

$$
R M_{t}^{\text {pre }}=\sum_{1 \leq i, j \leq 1 / h} q_{i j}^{\text {pre }} r_{t-1+i h} r_{t-1+j h}
$$

where

$$
q_{i j}^{p r e}=\frac{12}{\theta \sqrt{N}} q_{i j}^{\Phi}-\frac{6}{\theta^{2} N} q_{i j}^{a l l}
$$

and

$$
q_{i j}^{\Phi}=\sum_{l=0}^{N-k} \delta_{l+1 \leq i \leq l+k} \delta_{l+1 \leq j \leq l+k} \Phi\left(\frac{i-l}{k}\right) \Phi\left(\frac{j-l}{k}\right) .
$$

$\delta_{a \leq b \leq c}$ is the indicator function equal to 1 when $a \leq b \leq c$ and 0 otherwise.

Proof:

$$
R M_{t}^{\text {pre }}=\frac{12}{\theta \sqrt{N}} \sum_{i=0}^{N-k}\left\{\sum_{j=1}^{k} \Phi\left(\frac{j}{k}\right) r_{t-1+(i+j) h}\right\}^{2}-\frac{6}{\theta^{2} N} \sum_{i=1}^{N} r_{t-1+i h}^{2}
$$




$$
\begin{aligned}
& =\frac{12}{\theta \sqrt{N}} \sum_{i=0}^{N-k}\left(\sum_{l, m=1}^{k} \Phi\left(\frac{l}{k}\right) \Phi\left(\frac{m}{k}\right) r_{t-1+(i+l) h} r_{t-1+(i+m) h}\right)-\frac{6}{\theta^{2} N} \sum_{i=1}^{N} r_{t-1+i h}^{2} \\
& =\frac{12}{\theta \sqrt{N}} \sum_{i=0}^{N-k}\left(\sum_{I, J=1+i}^{k+i} \Phi\left(\frac{I-i}{k}\right) \Phi\left(\frac{J-i}{k}\right) r_{t-1+I h} r_{t-1+J h}\right)-\frac{6}{\theta^{2} N} \sum_{i=1}^{N} r_{t-1+i h}^{2} \\
& =\frac{12}{\theta \sqrt{N}} \sum_{i=0}^{N-k}\left(\sum_{I, J=1}^{N} \delta_{1 \leq I-i \leq k} \delta_{1 \leq J-i \leq k} \Phi\left(\frac{I-i}{k}\right) \Phi\left(\frac{J-i}{k}\right) r_{t-1+I h} r_{t-1+J h}\right)-\frac{6}{\theta^{2} N} \sum_{i=1}^{N} r_{t-1+i h}^{2} \\
& =\frac{12}{\theta \sqrt{N}} \sum_{I, J=1}^{N}(\underbrace{\sum_{i=0}^{N-k} \delta_{1 \leq I-i \leq k} \delta_{1 \leq J-i \leq k} \Phi\left(\frac{I-i}{k}\right) \Phi\left(\frac{J-i}{k}\right)}_{=q_{I J}^{\Phi}}) r_{t-1+I h} r_{t-1+J h}-\frac{6}{\theta^{2} N} \sum_{i=1}^{N} r_{t-1+i h}^{2} \\
& =\frac{12}{\theta \sqrt{N}} \sum_{I, J=1}^{N} q_{I J}^{\Phi} r_{t-1+I h} r_{t-1+J h}-\frac{6}{\theta^{2} N} \sum_{I, J=1}^{N} q_{I J}^{a l l} r_{t-1+I h} r_{t-1+J h} \\
& =\sum_{I, J=1}^{N} \underbrace{\left(\frac{12}{\theta \sqrt{N}} q_{I J}^{\Phi}-\frac{6}{\theta^{2} N} q_{I J}^{a l l}\right)}_{=q_{I J}^{\text {pre }}} r_{t-1+I h} r_{t-1+J h} \\
& =\sum_{I, J=1}^{N} q_{I J}^{p r e} r_{t-1+I h} r_{t-1+J h}
\end{aligned}
$$

In the next section, I show how such a quadratic-form representation of the pre-averaging estimator could be used to evaluate its performance in terms of forecasting.

\section{Application: Volatility Forecasting}

In order to assess the forecasting performance of the pre-averaging estimator $R M_{t}^{\text {pre }}$, I use a Mincer and Zarnowitz (1969) regression. More precisely, a high $R^{2}$ from the regression of $I V_{t+1}$ onto a constant and $R M_{t}^{\text {pre }}$ is considered as evidence of good performance of $R M_{t}^{p r e}$ as a forecast for $I V_{t+1}$. Formally,

$$
R^{2}\left(I V_{t+1}, R M_{t}^{\text {pre }}\right)=\frac{\operatorname{Cov}\left[I V_{t+1}, R M_{t}^{\text {pre }}\right]^{2}}{\operatorname{Var}\left[I V_{t+1}\right] \operatorname{Var}\left[R M_{t}^{\text {pre }}\right]} .
$$

The quadratic-form representation introduced in Section 3 is used to derive the expressions of the numerator and denominator of the aforementioned $R^{2}$ as follows:

$$
\begin{gathered}
\operatorname{Cov}\left[I V_{t+1}, R M_{t}^{\text {pre }}\right]=\sum_{1 \leq i, j \leq 1 / h} q_{i j}^{\text {pre }} \operatorname{Cov}\left[I V_{t+1}, r_{t-1+i h} r_{t-1+j h}\right] \\
\operatorname{Var}\left[R M_{t}^{\text {pre }}\right]=E\left[\left(R M_{t}^{\text {pre }}\right)^{2}\right]-E\left[R M_{t}^{\text {pre }}\right]^{2} \\
E\left[\left(R M_{t}^{\text {pre }}\right)^{2}\right]=\sum_{1 \leq i, j, k, l \leq 1 / h} q_{i j}^{\text {pre }} q_{k l}^{\text {pre }} E\left[r_{t-1+i h} r_{t-1+j h} r_{t-1+k h} r_{t-1+l h}\right]
\end{gathered}
$$




$$
E\left[R M_{t}^{\text {pre }}\right]=\sum_{1 \leq i, j \leq 1 / h} q_{i j}^{\text {pre }} E\left[r_{t-1+i h} r_{t-1+j h}\right]
$$

In order to derive a closed-form formula for the $R^{2}$, the expressions of the moments $\operatorname{Cov}\left[I V_{t+1}, r_{t-1+i h} r_{t-1+j h}\right], \operatorname{Var}\left[I V_{t+1}\right], E\left[r_{t-1+i h} r_{t-1+j h} r_{t-1+k h} r_{t-1+l h}\right]$ and $E\left[r_{t-1+i h} r_{t-1+j h}\right]$ are needed. Indeed, the ESV representation developed by Meddahi (2001) provides a theoretical framework to derive such expressions. If I assume that the volatility is driven by a single-state variable $f_{t}$, the spot volatility takes the form

$$
\sigma_{t}^{2}=\sum_{n}^{p} a_{n} P_{n}\left(f_{t}\right)
$$

where the integer $p$ may be infinite. I assume the normalization $P_{0}\left(f_{t}\right)=1$. The latent state variable evolves as $f_{t}=m\left(f_{t}\right) d t+\sqrt{v\left(f_{t}\right)} d W_{t}^{f}$, where the $W_{t}^{f}$ Brownian motion is independent of the $W_{t}$ Brownian motion driving the frictionless price. Furthermore, the $a_{n}$ coefficients are real numbers and the $P_{n}\left(f_{t}\right)$ denote the eigenfunctions of the infinitesimal generator associated with $f_{t}$. In particular, $P_{n}\left(f_{t}\right)$ are orthogonal and centered at zero,

and follow first-order autoregressive processes,

$$
\begin{gathered}
E\left[P_{n}\left(f_{t}\right) P_{j}\left(f_{t}\right)\right]=0 \\
E\left[P_{n}\left(f_{t}\right)\right]=0
\end{gathered}
$$

$$
\forall l>0, n>0, E\left[P_{n}\left(f_{t+l}\right) \mid f_{\tau}, \tau \leq t\right]=\exp \left(-\gamma_{n} l\right) P_{n}\left(f_{t}\right)
$$

where $\left(-\gamma_{n}\right)$ denotes the corresponding eigenvalues.

In Proposition 2.1 of Andersen et al. (2011), the authors derive the expressions of the necessary moments to compute the Mincer-Zarnowitz $R^{2}$ under i.i.d. noise contamination. And, the expression of $\operatorname{Var}\left[I V_{t+1}\right]$ is provided in Equation (3.8) of Andersen et al.'s paper.

\section{Conclusion}

In this paper, I provide a mathematically tractable quadratic-form representation of the pre-averaging estimator introduced in Jacod et al. (2009). Building on Andersen et al. (2011), I show how such a representation could be used to theoretically examine the volatility forecasting performance for the pre-averaging estimator.

\section{References}

Ait-Sahalia, Y., P.A. Mykland and L. Zhang (2011), Ultra high frequency volatility estimation with dependent microstructure noise, Journal of Econometrics, 160, 160-175.

Andersen, T.G., T. Bollerslev and N. Meddahi (2006), Realized volatility forecasting and market microstructure noise, Working paper. Montreal University.

Andersen, T.G., T. Bollerslev and N. Meddahi (2004), Analytic Evaluation of Volatility Forecasts, International Economic Review, 45, 1079-1110.

Andersen, T.G., T. Bollerslev, and N. Meddahi (2011), Realized volatility forecasting and market microstructure noise, Journal of Econometrics, 160, 220-234.

Barndorff-Nielsen, O.E., P.R. Hansen, A. Lunde and N. Shephard (2008), Designing realized kernels to measure the ex-post variation of equity prices in the presence of noise, Econometrica, 76, 6, 1481-1536.

Barndorff-Nielsen, O.E., P.R. Hansen, A. Lunde and N. Shephard (2011), Multivariate realised kernels: Consistent positive semi-definite estimators of the covariation of equity prices with noise and non-synchronous trading, Journal of Econometrics, $162,149-169$. 
Barndorff-Nielsen, O.E. and N. Shephard (2002), Econometric Analysis of Realized Volatility and Its Use in Estimating Stochastic Volatility Models, Journal of the Royal Statistical Society. Series B (Statistical Methodology), 64, 2, 253-280.

Jacod, J. (1994), Limit of random measures associated with the increments of a Brownian semimartingale, Tech. Rep. Universite Paris VI.

Jacod, J., Y. Li, P. Mykland, M. Podolskij and M. Vetter (2009), Microstructure noise in the continuous case: The pre-averaging approach, Stochastic Processes and their Applications, 119, 2249-2276.

Kalnina, I. and O. Linton (2008), Estimating quadratic variation consistently in the presence of endogenous and diurnal measurement error, Journal of Econometrics, 147, 47-59.

Meddahi, N. (2001), An Eigenfunction Approach for Volatility Modeling, CIRANO working paper, 2001s-70.

Mincer, J. and V. Zarnowitz (1969), The Evaluation of Economic Forecasts, J. Mincer (ed.), Economic Forecasts and Expectations, New York: National Bureau of Economic Research.

Zhang, L. (2006), Efficient estimation of stochastic volatility using noisy observations: A multi-scale approach, Journal of the American Statistical Association, 100, 1394-1411.

Zhang, L., P.A. Mykland and Y. A“it-Sahalia (2005), A Tale of Two Time Scales: Determining Integrated Volatility with Noisy High-Frequency Data, Journal of the American Statistical Association, 100, 1394-1411.

Sizova, N. (2011), Integrated variance forecasting: Model based vs. reduced form, Journal of Econometrics, 162, $294-311$. 